\title{
Il Linguaggio Be-Bop e gli Accordi di Settima di Prima Specie [The Be-Bop Language and The Dominant Seventh Chords]
}

\author{
Authors: $\quad$ Carmine Cataldo \\ Submitted: $\quad$ 17. October 2017 \\ Published: $\quad$ 17. October 2017 \\ Volume: 4 \\ Issue: $\quad 4$ \\ Affiliation: Independent Researcher, Jazz Pianist and Composer, PhD in \\ Mechanical Engineering, Battipaglia (SA), Italy \\ Keywords: Improvvisazione Jazz, Linguaggio Be-Bop, Accordi di Settima di \\ Dominante, Triadi Maggiori [Jazz Improvisation, Be-Bop \\ Language, Dominant Seventh Chords, Major Triads] \\ DOI: $\quad 10.17160 /$ josha.4.4.340
}




\title{
Il Linguaggio Be-Bop e gli Accordi di Settima di Prima Specie
}

\author{
Carmine Cataldo \\ Jazz Pianist and Composer, PhD in Mechanical Engineering, Battipaglia (SA), Italy \\ Email: catcataldo@hotmail.it
}

\begin{abstract}
English)
This article represents a translated and revised version of the paper "The Art of Improvising: the Be-Bop Language and the Dominant Seventh Chords". In this paper we exclusively discuss the improvisation techniques concerning the Dominant Seventh Chords. The reader, who basically is not required to possess any particular jazz background, is immediately led to speaking, as it were, by resorting to the Be-Bop language. On this subject, it is fundamental to underline how the whole dissertation is carried out in terms of sentences and words. The method herein proposed is very simple and intuitive. We start from a specific sentence, regarded as fundamental, built upon the Major triad. By exploiting the fundamental sentence, several further sentences can be instantly deduced. The sentences so obtained can be easily cut, modified, by adding new simple words, combined with each other and finally enriched.
\end{abstract}

\section{Keywords (English)}

Jazz Improvisation, Be-Bop Language, Dominant Seventh Chords, Major Triads.

\begin{abstract}
In questo articolo vengono discusse, in via esclusiva, le tecniche d'improvvisazione relative agli Accordi di Settima di Dominante. Il lettore, cui di fatto non è richiesto il possesso d'alcun background di carattere jazzistico, è subito portato ad esprimersi, per così dire, adoperando il Linguaggio Be-Bop. Il metodo è semplice e intuitivo. Si parte dalla presentazione d'una "Frase Fondamentale", costruita sulla Triade Maggiore, dalla quale vengono istantaneamente dedotte ulteriori proposizioni. Le frasi così ottenute vengono successivamente modificate, effettuando semplici "tagli" ovvero aggiungendo nuove "parole", e tra di loro variamente combinate.
\end{abstract}

\section{Keywords}

Improvvisazione Jazz, Linguaggio Be-Bop, Accordi di Settima di Dominante, Triadi Maggiori.

\section{Introduzione}

In questa sede vengono trattati, in via esclusiva, gli Accordi di Settima di Dominante (Prima Specie). $\mathrm{Si}$ parte da una "Frase Fondamentale", costruita intorno alla Triade Maggiore, dalla quale scaturiscono immediatamente tre proposizioni. Le frasi così ottenute possono essere agevolmente tagliate, variamente modificate, adoperando nuove parole (Wise, 1983) ovvero imponendo banali estensioni, a successivamente combinate. 
La peculiarità del metodo consiste, principalmente, nel fatto che l'attenzione dell'improvvisatore è costantemente mantenuta sulla triade. A tal proposito, anticipiamo come la suddetta peculiarità possa perdere apparentemente rilievo nel momento in cui vengano introdotte settima (minore) e nona. L'integrità del metodo, tuttavia, può essere serenamente preservata focalizzando l'attenzione non più sulla triade maggiore (relativa all'accordo considerato), bensì su quella minore distante una quarta giusta discendente (dalla tonica dell'accordo considerato). Ad ogni modo, tale aspetto verrà esaustivamente discusso nella sezione "Osservazioni Finali a Conclusioni”.

\section{Il Metodo}

\subsection{Presentazione della "Frase Fondamentale"}

Sebbene il metodo debba essere praticato e padroneggiato in tutte e 12 le tonalità, in quest'articolo, per ovvie motivazioni, ci limiteremo a discutere, in via esclusiva, l'accordo $C 7$.

Precisando come tale pratica rappresenti tutto fuorchè una novità (Wise, 1983), condurremmo l'intera discussione in termini di frasi e parole. Prima d'iniziare, vale la pena sottolineare come l'unico background richiesto consista, de facto, nella mera conoscenza delle triadi.

La frase fondamentale dalla quale occorre partire è mostrata nel sottostante Pentagramma (1):

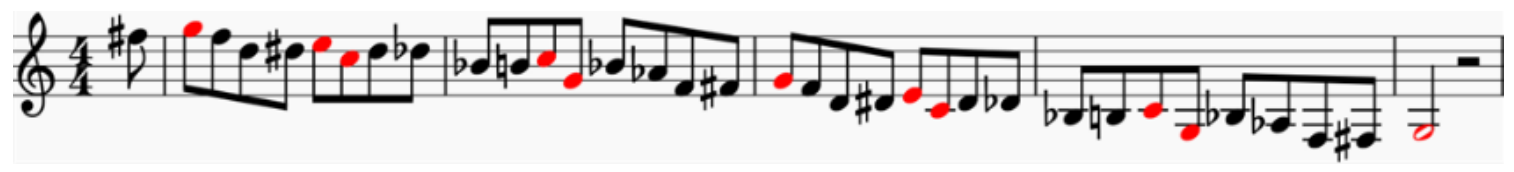

Nel Pentagramma (1), come del resto nella totalità dei pentagrammi inclusi nel presente articolo (al netto di una singola eccezione), le note cordali (da intendersi come quelle costituenti la triade) sono rappresentate in rosso.

La frase fondamentale, estesa intenzionalmente lungo due ottave adiacenti, consente di suonare, in una volta, la totalità delle tensioni alterate dell'accordo (b13, \#11, \#9, b9).

\subsection{Focalizzare l'Attenzione sulla Triade Maggiore}

La frase fondamentale può essere evidentemente eseguita, considerando una semplice traslazione, partendo da ognuna delle note cordali.

È fondamentale sottolineare come, nel riprodurre tanto la frase fondamentale quanto le proposizioni derivate, l'attenzione debba essere costantemente focalizzata sulla triade maggiore (stato fondamentale e rivolti).

Dal Pentagramma (1), sfruttando la traslazione ed effettuando dei banali tagli, otteniamo immediatamente le tre utilissime frasi sottostanti:

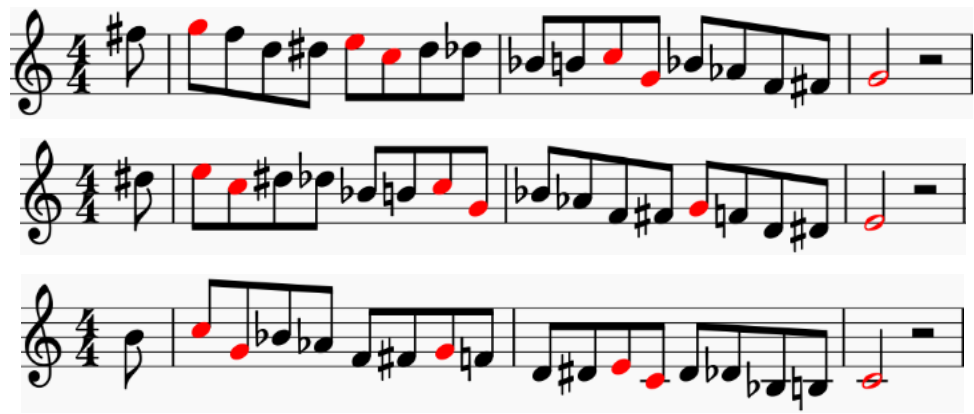


L'approccio ascendente (ovviamente cromatico) che appare nelle anacrusi è da ritenersi opzionale. Sebbene l'argomento non venga in questa sede approfondito, è interessante notare come le frasi proposte nei Pentagrammi (2), (3) e (4), si prestino agevolmente a svariati spostamenti metrici.

A questo punto, vale la pena dedicarsi alla pratica del sottostante esercizio, tanto semplice quanto utile, basato sulla triade maggiore e gli approcci (cromatici) ascendenti:

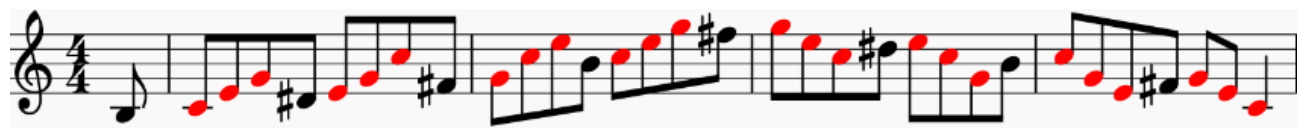

Dai Pentagrammi (2), (3) e (4) (il primo dei quali trasposto all'ottava inferiore), imponendo un banale spostamento metrico (traslazione pari ad un movimento) e fruttando il precedente esercizio, otteniamo le tre frasi sottostanti:
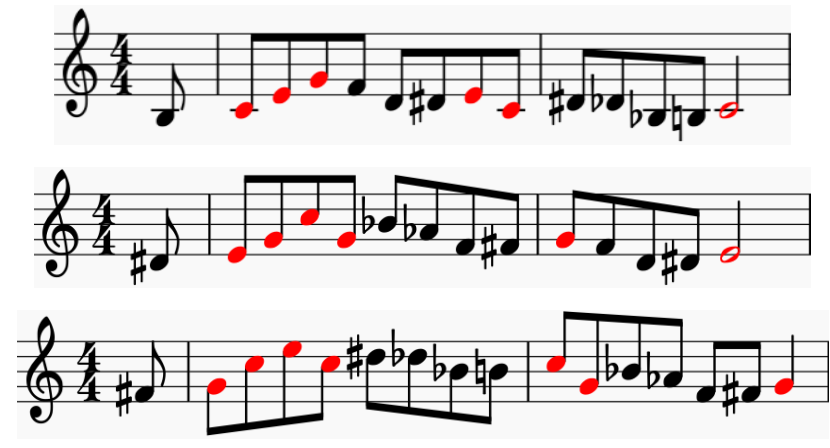

Siamo già in grado di combinare le semplici proposizioni finora ottenute, al fine di generarne altre assai più articolate (Baker, 1988a, 1988b, 1988c, 1988d; Wise, 1983).

Ad esempio, dai Pentagrammi (6), (7) e (8) (quest'ultimo trasposto all'ottava superiore), possiamo dedurre la seguente frase:

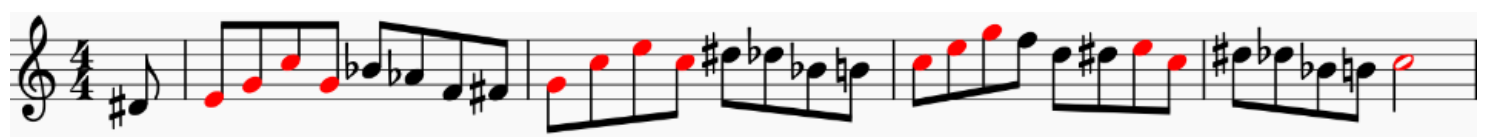

Dedichiamoci ora alla pratica del sottostante esercizio, basato sul concetto di approccio "misto":

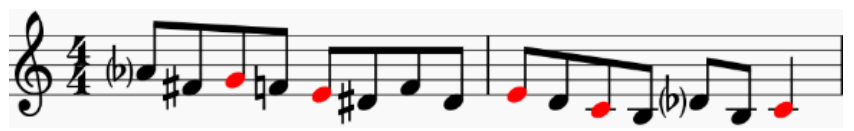

La frase precedente conferisce all'improvvisazione un "mood" decisamente "cromatico": tale effetto è amplificato dall'adozione degli accidenti opzionali riportati in parentesi.

Ovviamente, in linea con quanto evidenziato all'inizio del paragrafo corrente, il musicista deve aver cura, nell'eseguire l'esercizio proposto nel Pentagramma (10), di focalizzare la propria attenzione sulla triade maggiore.

Naturalmente, possiamo continuare a combinare frasi, ora con un "ingrediente" addizionale.

Ad esempio, dai Pentagrammi (3) e (10) (quest'ultimo trasposto all'ottava superiore), otteniamo immediatamente:

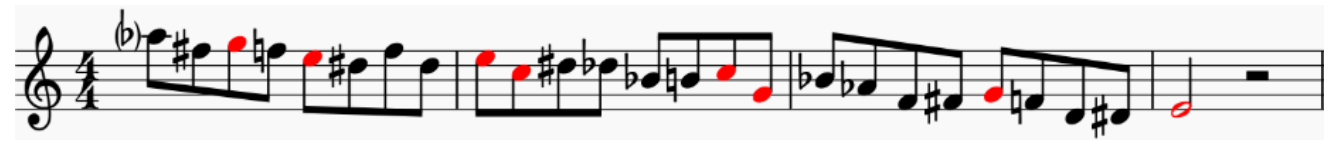


Consideriamo adesso la sottostante nuova parola, nulla più che un ponte cromatico bidirezionale (da percorrersi, dunque, in entrambi versi, ascendendo e discendendo) tra la quinta e la sesta:

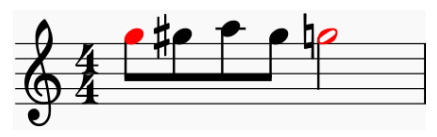

L'attenzione dell'esecutore, naturalmente, dev'essere focalizzata in via esclusiva sulla quinta. Sfruttando la parola appena introdotta, è possibile dedurre un enorme quantitativo di nuove frasi. Ad esempio, dal Pentagramma (2), con un'estensione alla terza, e dal Pentagramma (12), otteniamo:

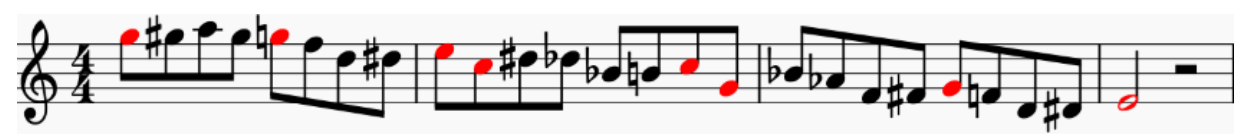

\subsection{Introduzione della Settima}

Consideriamo la seguente nuova parola, nient'altro che un semplice ponte cromatico discendente tra la settima minore (rappresentata in verde) e la quinta:

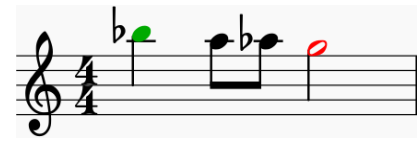

Dal Pentagramma (2), traslato ed esteso alla terza, e dal Pentagramma (14), otteniamo:

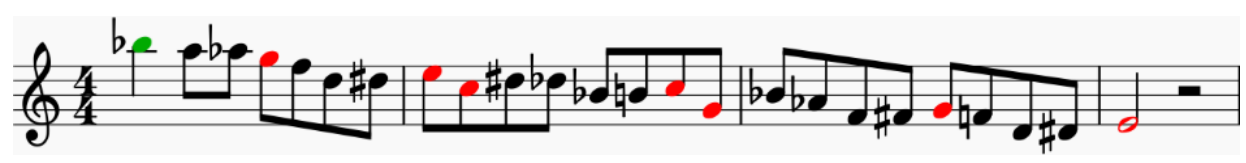

Dalla giustapposizione dei Pentagrammi (12) e (14), otteniamo la sottostante nuova parola:

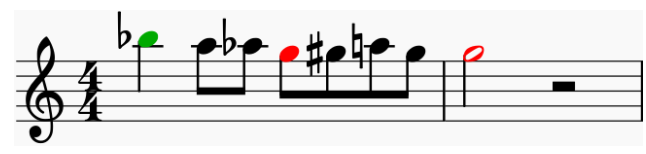

Sfruttando la parola appena ottenuta, dal Pentagramma (2), imponendo la solita estensione alla terza, deduciamo agevolmente la seguente frase:

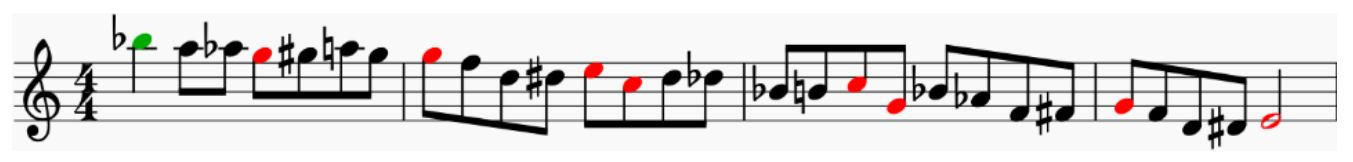

Adesso, accantoniamo per un attimo la settima.

La frase presentata nel Pentagramma (2) può essere modificata "tagliando corto". Una volta raggiunta la terza, anziché proseguire nel rispetto della frase, costruiamo un ponte cromatico, semplice e diretto, verso la tonica:

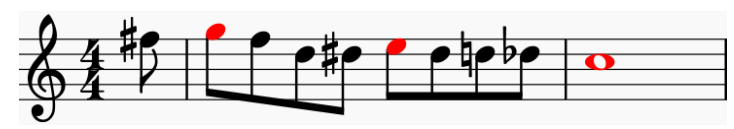

Vale la pena evidenziare come la frase precedente possa essere serenamente eseguita considerando Fmaj7 in ultima battuta. 
Dai Pentagrammi (12) e (18) otteniamo immediatamente:

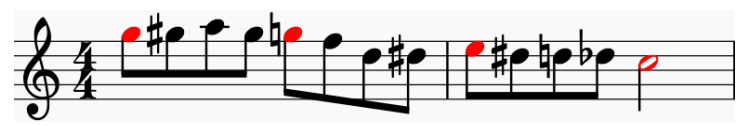

Recuperando la settima, dai Pentagrammi (14) e (18) deduciamo il seguente:

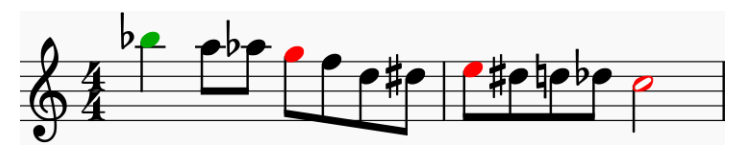

Similmente, dai Pentagrammi (16) e (18) otteniamo:

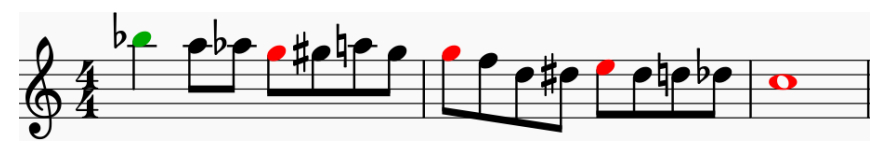

La frase appena dedotta può essere serenamente suonata sulla progressione armonica G-7/C7/Fmaj7. La frase nel Pentagramma (2) può essere ulteriormente modificata a mezzo d'una "deviazione". Una volta raggiunta la terza, anziché riprodurre la frase nella maniera in cui essa è stata inizialmente concepita, si devia lungo la ben nota Scala "Dominante Be-Bop", sino a raggiungere la settima:

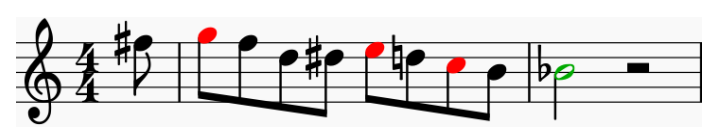

Dai Pentagrammi (12) e (22) abbiamo:

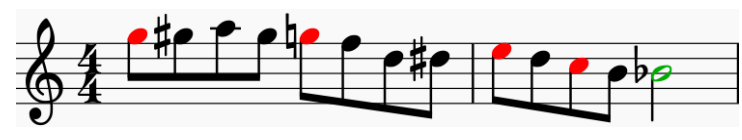

Dai Pentagrammi (14) e (22) abbiamo:

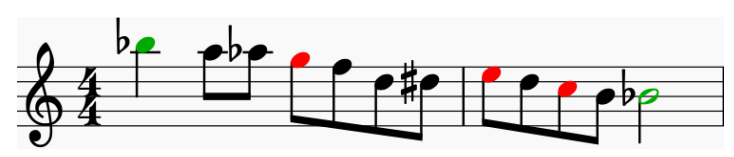

Dai Pentagrammi (16) e (22) abbiamo:

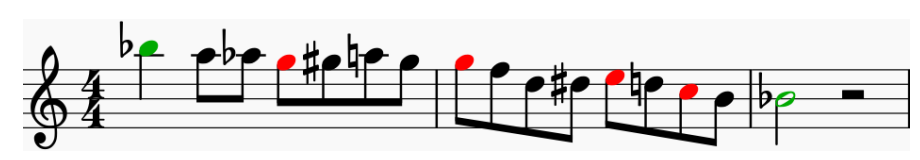

Estendendo la proposizione precedente lungo due ottave consecutive, otteniamo una nuova frase, in cui il ruolo della tonica è ora interpretato, per così dire, dalla settima minore:

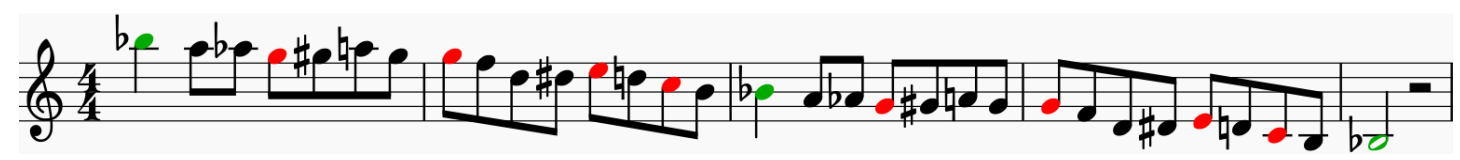

Dalla frase appena ottenuta, adoperando una procedura del tutto simile a quella sfruttata al fine di dedurre i Pentagrammi (2), (3) e (4), è possibile ricavare tre proposizioni semplici, omesse per brevità, che possono essere ovviamente modificate seguendo una metodologia che, a questo punto, dovrebbe risultare abbastanza chiara. 
Evidentemente, siamo ora in grado di comporre un numero significativo di nuove frasi, così coprendo una larga fetta del cosiddetto "Vocabolario" Be-Bop (Baker, 1988a, 1988b, 1988c; Wise 1983).

Ad esempio, sovrapponendo opportunamente i Pentagrammi (7), (22) e (25) (il primo trasposto all'ottava superiore, l'ultimo trasposto all'ottava inferiore ed arrestato una volta raggiunta la terza), otteniamo agevolmente:

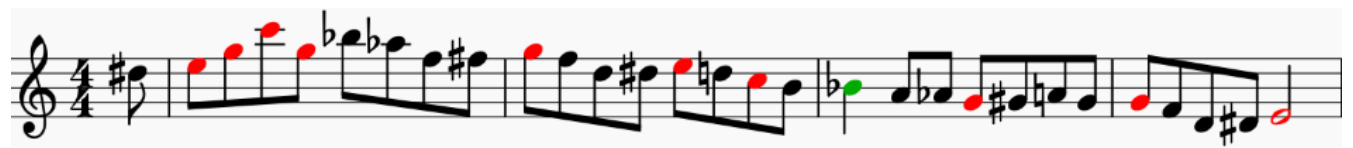

Ovviamente, ulteriori modificazioni, non importa quanto significative, consentono d'incrementare notevolmente il numero delle possibilità. A tal proposito, basti pensare che il ponte cromatico introdotto nel Pentagramma (18) può anche essere bidirezionale. Alla luce di quanto appena osservato, sfruttando la deviazione introdotta nel Pentagramma (22), possiamo agevolmente dedurre la sottostante utilissima frase:

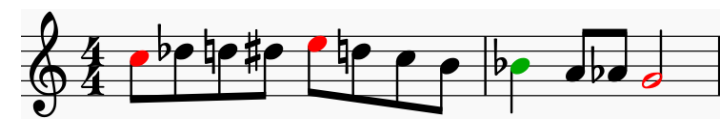

Dai Pentagrammi (9) e (28), agendo nel solito modo, abbiamo:

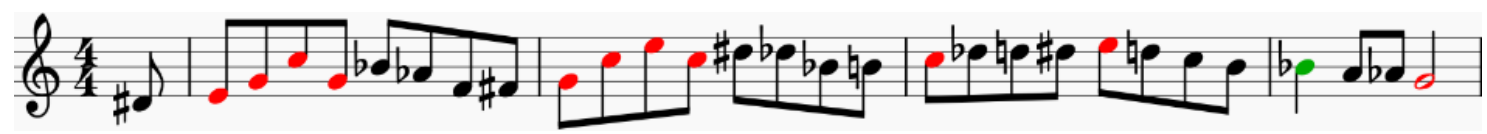

\subsection{Introduzione della Nona}

Il metodo brevemente presentato in quest'articolo dovrebbe oramai risultare sufficientemente chiaro: pertanto, per amore di sintesi, ometteremo la scrittura di singole parole nella sezione corrente.

Anzitutto, è evidentemente possibile la costruzione di un semplice ponte cromatico discendente tra la nona e la settima (entrambe rappresentate in verde). Sfruttando tale procedura, dal Pentagramma (25), trasposto all'ottava inferiore ed esteso alla quinta, possiamo dedurre la seguente frase:

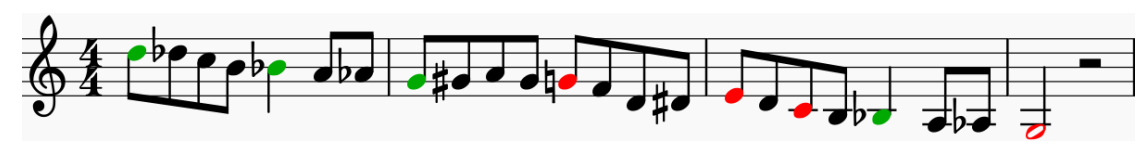

È naturalmente contemplabile la costruzione di ponti cromatici bidirezionali: il più semplice, ovviamente, è quello che coinvolge nona e decima (la terza all'ottava superiore).

Dai Pentagrammi (21) e (30), ad esempio, otteniamo:

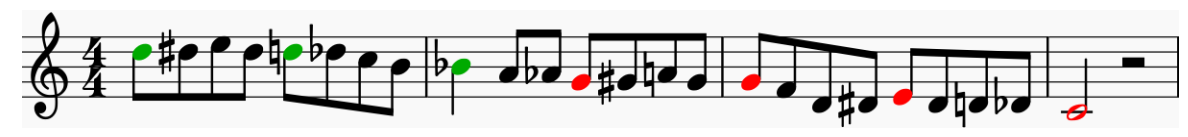

Il cromatismo bidirezionale di maggior interesse è quelle che coinvolge settima minore e nona. Ad esempio, dal Pentagramma (30) possiamo immediatamente dedurre la sottostante frase:

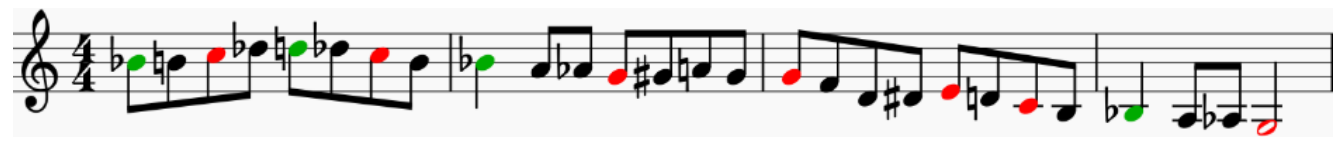




\subsection{Come "Arricchire" una Frase (cenni brevissimi)}

Non appena l'improvvisatore acquisisce sufficiente dimestichezza con il metodo, può avere finalmente inizio una fase di "arricchimento". Sebbene la procedura non venga in questa sede affrontata, possiamo sinteticamente affermare come il modo più semplice per arricchire una frase consista nell'utilizzo di "ripetizioni”" ed "oscillazioni diatoniche" (Garland, 1999; Kelly, 2013; Powell, 1998, 2002). Un semplice esempio di arricchimento, interamente basato sulla frase nel Pentagramma (30), è di seguito riportato:

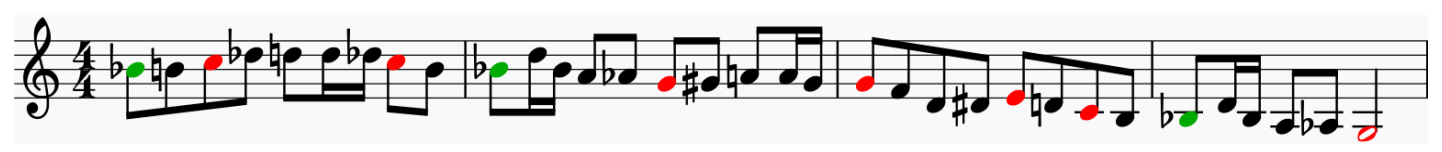

\section{Osservazioni Finali e Conclusioni}

Nel Linguaggio Be-Bop, gli Accordi di Settima di Dominante sono spesso sostituiti con Accordi Diminuiti (Parker, 1978) costruiti su terza, quinta, settima (minore) e nona. A tal proposito, si rivela utilissimo il seguente esercizio:

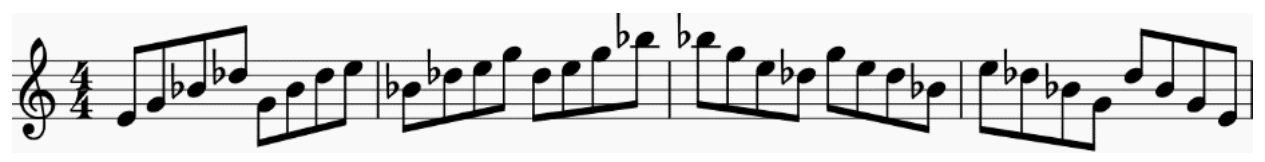

Sfruttando la sostituzione sopra menzionata (Levine, 2009), è possibile modificare ulteriormente le frasi finora ottenute. Proponiamo, dunque, una coppia di utilisimi esempi, in cui la nona bemolle è intenzionalmente rappresentata in arancione.

Dal Pentagramma (25), trasposto all'ottava inferiore, otteniamo:

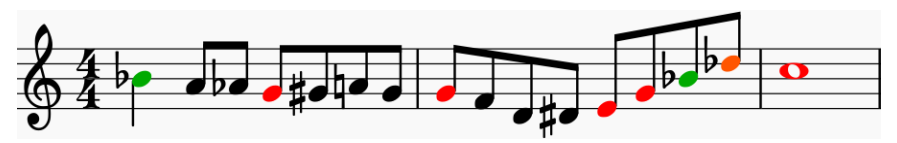

Dal Pentagramma (30), tagliato e privato del cromatismo bidirezionale sulla quinta, otteniamo:

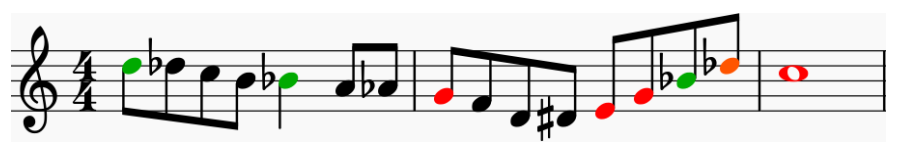

Le ultime due frasi possono essere evidentemente suonate sulla progressione $G-7 / C 7 / F m a j 7$. La cosa costituisce tutto fuorchè una coincidenza: al contrario, abbiamo finalmente l'opportunità di discutere qualitativamente il motivo per cui, nonostante l'introduzione di note non cordali (settima minore e nona), il metodo possa essere ancora considerato come basato esclusivamente sulle triadi. Nella prima battuta del Pentagramma (36), ad esempio, l'attenzione dell'improvvisatore dev'essere focalizzata non già sulla triade maggiore di $C$, bensì su quella minore di $G$. In certa misura, infatti, è lecito affermare come $C 7$ possa essere "trattato" come se fosse $G-7$ (i due accordi sono "parenti di secondo grado", siccome entrambi in relazione diatonica con $E-7 b 5)$. Di conseguenza, al netto di rare eccezioni, l'improvvisatore può serenamente suonare su $C 7$ la quasi totalità delle frasi adatte a $G-7$ (gli accordi di seconda specie non vengono analizzati presente articolo), e vice versa, in tal modo ampliando considerevolmente il proprio linguaggio. 
Infine, per quanto ovvio, è doveroso specificare come il metodo sinteticamente presentato in questo articolo costituisca nulla più che un'introduzione, tra l'altro assai semplificata, al Linguaggio BeBop. Non a caso, è stata intenzionalmente evitata l'analisi delle metodologie d'impiego di alcune scale fondamentali (esafoniche, come quella a Toni Interi, eptafoniche, come la Super-Locria, octofoniche, come la Semitono-Tono), dei numerosi "pattern di pubblico dominio" (assimilabili ad espressioni idiomatiche ovvero a "riferimenti eruditi") (Coker et al.,1982; Nelson, 2010), delle sostituzioni armoniche al tritono (Levine, 2009).

\section{Ringraziamenti}

Questo articolo è dedicato a mia madre, Giuseppina, che mi ha sempre incoraggiato a suonare il piano con passione e devozione. Ringrazio gli amici Francesco D'Errico, Giulio Martino, e Sandro Deidda, eccellenti jazzisti italiani e stimati docenti presso il Conservatorio di Salerno, per i loro preziosi suggerimenti.

\section{Riferimenti}

Baker, D. (1988a). How to Play Bebop (Volume 1). Los Angeles, CA: Alfred Publishing Co. Inc.

Baker, D. (1988b). How to Play Bebop (Volume 2). Los Angeles, CA: Alfred Publishing Co. Inc.

Baker, D. (1988c). How to Play Bebop (Volume 3). Los Angeles, CA: Alfred Publishing Co. Inc.

Baker, D. (1988d). Jazz Improvisation. Los Angeles, CA: Alfred Publishing Co. Inc.

Coker, J., Casale, J., \& Campbell, G. (1982). Patterns for Jazz. Los Angeles, CA: Alfred Publishing Co. Inc.

Dobbins, B. (2010). Jazz Arranging and Composing - L'Approccio Lineare (Italian Ed. by Roberto Spadoni). Italy: Volontè \& Co.

Garland, R. (1999). The Jazz Piano Solos of Red Garland (by Tony Genge). Houston, TX: Houston Publishing. Kelly, W. (2013). The Wynton Kelly Collection: 25 Solo Transcriptions (by Michael Miller). New Albany, IN: Jamey Aebersold Jazz.

Levine, M. (2009). The Jazz Theory Book (Italian Edition by F. Jegher). Milan, IT: Curci Jazz.

Nelson, O. (2010). Patterns for Improvisation. New Albany, IN Jamey Aebersold Jazz.

Parker. C. (1978). Charlie Parker Omnibook. Los Angeles, CA: Atlantic Music Corporation.

Powell, B. (1998), Bud Powell Classics (Artist Transcriptions). Milwaukee, WI: Hal · Leonard.

Powell, B. (2002). The Bud Powell Collection: Piano Transcriptions (Artist Transcriptions). Milwaukee, WI: Hal $\cdot$ Leonard.

Wise. L. (1983). Bebop Bible - The Musicians Dictionary of Melodic Lines. United States: REH Publications.

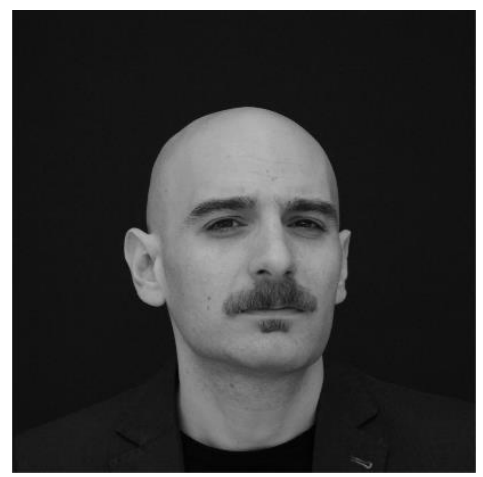

Author Info

Carmine Cataldo

Independent Researcher, Battipaglia (SA), Italy

$\mathrm{PhD}$ in Mechanical Engineering

Jazz Pianist and Composer

Art Director and Resident Pianist at "Bar Capri", Battipaglia (SA), Italy

Email: catcataldo@hotmail.it

ResearchGate Profile:

https://www.researchgate.net/profile/Carmine Cataldo

LinkedIn Profile:

https://it.linkedin.com/in/carmine-cataldo-3ab097122 


\section{Curriculum Vitae (excerpt)}

Carmine Cataldo was born on 13 February 1979 in Battipaglia (Salerno - Italy).

In 2004, he graduates from the University of Salerno in Mechanical Engineering; the same year, he passes the qualifying examination to the engineers register. In 2008, he obtains, from the University of Salerno, a $\mathrm{PhD}$ in Mechanical Engineering, with a final dissertation based upon the analytical modelling of the extrusion and stretching processes targeted at thermoplastic polymers. During PhD research work, he mainly focuses on heat treating of steels and iron based alloys, mechanical characterization of unconventional and composite materials, innovative technologies for welded and glued joints, treatments finalized to increase the surface tension of polymeric films, the application of fuzzy logic in order to adjust the extrusion die during the film casting process and rapid prototyping by laser sintering. Currently, he is particularly interested in alternative cosmology and special relativity. His main interest lies in the attempt to preserve the validity of notions, considered as outdated, by assigning a different meaning, coherently with the phenomenological reality, to equations usually classified as relativistic. He is a member of the editorial board of the journal "Research and Reviews: Journal of Pure and Applied Physics"

Carmine Cataldo is also a jazz pianist and composer.

He has been resident pianist and art director at music club "Bar Capri", in Battipaglia (Italy), since 2004. In 2002 he ranks first in the International Jazz Competition "Baronissi Jazz Festival" (Emerging Musicians Category); moreover, he is appointed best young talent in the competition and awarded a scholarship for the Berklee Summer School at Umbria Jazz 2003. During the Berklee Clinics he studies Piano Improvisation (advanced level) with Russell Hoffmann, Ensemble Music with the guitarist Jim Kelly, Improvisation Techniques with the saxophonist Greg Badolato, Be-Bop Language with the trumpeter Jeff Stout, and attends special lectures with the renowned singer Bobby McFerrin and the legendary drummer Elvin Jones. In 2003 he ranks first in the International Jazz Competition "Baronissi Jazz Festival" (Professional Musicians Category). He has had the privilege of sharing the stage with several worldwide esteemed jazz musicians, such as Avishai Cohen (Baronissi Jazz Festival 2004), Stefano Bollani and Enrico Rava (Jazz Castello Lagopesole, 11th Edition). As a jazz pianist, he has cooperated with artists of the calibre of Alfonso Deidda, Antonio Onorato, Carla Marciano, Daniele Scannapieco, Giulio Martino, Jerry Popolo, Max Ionata, Pietro Condorelli, Sandro Deidda.

Carmine Cataldo is also a martial arts teacher.

He is appointed "Shifu" by his own master, Sifu Antonello Parisi (ITKAA), on 18 December 2016. $\mathrm{He}$ is a Black Belt in Shaolin Wing Chun (Superior Instructor, 4th Technical Level) and Combat Escrima Concept (Advanced Instructor, 3rd Technical Level). He has attended several seminars with renowned martial artists of the calibre of Grand Master Renè Latosa (Escrima Concepts), Sifu Maria Gröthe (Siu Lam Weng Chun), Sifu Lin Xiang Fuk (Black Flag - Hek Ki Boen Eng Chun), Master Marco Mattioni (Escrima and Wing Tsun), Master Aldo Chiari (Muay Thai Boran). 